\title{
Prey-predator model in drainage system with migration and harvesting
}

https://doi.org/10.1515/msds-2021-0131

Received March 9, 2021; accepted May 3, 2021

\begin{abstract}
In this paper, we consider a prey-predator model with a reserve region of predator where generalist predator cannot enter. Based on the intake capacity of food and other factors, we introduce the predator population which consumes the prey population with Holling type-II functional response; and generalist predator population consumes the predator population with Beddington-DeAngelis functional response. The densitydependent mortality rate for prey and generalist predator are considered. The equilibria of proposed system are determined. Local stability for the system are discussed. The environmental carrying capacity is considered as a bifurcation parameter to evaluate Hopf bifurcation in the neighbourhood at an interior equilibrium point. Here the fishing effort is used as a control parameter to harvest the generalist predator population of the system. With the help of this control parameter, a dynamic framework is developed to investigate the optimal utilization of resources, sustainability properties of the stock and the resource rent. Finally, we present a numerical simulation to verify the analytical results, and the system is analyzed through graphical illustrations. The main findings with future research directions are described at last.
\end{abstract}

Keywords: Prey-predator system; Hopf bifurcation; Harvesting; Local and Global stability; Optimal control

MSC: 92B05; 34C23

\section{Introduction}

Predation is an important factor in a food chain. In predation, predators may or may not kill their prey prior to feeding on them, but the act of predation often results in the death of its prey through consumption. Moreover, predation is an interaction between the species in which one species uses another species as food. Generally, successful predation leads to increase in a population size of predator and to decrease in a population size of prey ([1],[2],[3], [4]). In this way, predation controls a food chain as well as an ecological system. To describe this phenomenon, many researchers ([5],[6],[7]) have shown their interest in this field.

Holling-Tanner prey-predator model with Beddington-DeAngelis functional response including delay has been discussed by Jana and Roy [8]. Predator-prey system with prey-taxis has been considered by Qiu et al. [9]. Tripathi et al. [10] analyzed global dynamics and parameter identifiability in a predator-prey interaction model. Banerjee et al. [11] discussed a prey-predator model with Holling Type-III functional response. Chaotic behavior of predator-prey model with group defense and non-linear harvesting in prey has been discussed by Kumar and Kharbanda [12]. Abid et al. [13] discussed about optimality in selective harvesting predator-prey model with modified Leslie-Gower and Holling Type II. Harvesting is the process of catching or killing those substances which are used to prepare foods. To fill up the demand of human needs and balance the species for future harvesting, harvesting effort is very much essential to optimize economically. Clark [14] considered

\footnotetext{
Banani Roy: Department of Applied Mathematics with Oceanology and Computer Programming, Vidyasagar University, Midnapore-721102, West Bengal, India, E-mail: banani89roy@gmail.com

*Corresponding Author: Sankar Kumar Roy: Department of Applied Mathematics with Oceanology and Computer Programming, Vidyasagar University, Midnapore-721102, West Bengal, India, E-mail: sankroy2006@gmail.com; roysank@mail.vidyasagar.ac.in, Orcid Id: 0000-0003-4478-1534
} 
the harvesting of one species in a fishery consisting of two competing species. Mathematical modelling in harvesting of such fisheries were studied by Chaudhuri et al. [15], Roy and Roy [16], Kar and Ghosh [17]. A harvesting strategy for a prey-predator dynamical system was studied by Sen et al. [18].

A nature reserve is a protected area which is very important for an ecological system. It may be designated by government institutions in some countries or by private land owners. In natural reserve region, limited number of species lived, all species cannot enter. In last few decades, researchers ([19],[20]) have shown their interest to study prey-predator system in a nature reserve region. They considered only one species i.e., prey lives in a reserve region in their works. But, many cases, there are more than one species live in a reserve region and they are related with prey-predator relationship.

We consider here a real-life example on our proposed problem for showing the feasibility and effectiveness of this work. In geomorphology, drainage systems are the patterns formed by the streams, rivers, and lakes in a particular drainage basin. Mosquito larvae grow in drainage system from where different types of mosquitoes are born. They are the carrier of different types of harmful diseases like Dengue, Malaria, Meningitis etc. Nowadays, harmful diseases are attacking to human population. A large number of peoples is affected by these harmful diseases. Controlling of mosquito population is an essential measure to prevent the harmful diseases. Mosquito fishes (Gambezi) live and also cultivate in a drainage system to prevent the mosquito populations. Here, gambezi is considered as predator and mosquito larva is treated as prey. A drainage basin is an area of land where all surface water from drainage system usually fall into the basin, as well as the water connects another body of water, such as a river, lake, reservoir, estuary, wetland, sea or ocean. Fishes like catfish lives in the other body of water like river, lake, reservoir etc. Catfish is considered here as generalist predator, which consumes mosquito fishes for their diet but catfish does not enter in drainage system. For this reason, drainage system is here the reserve region for the mosquito fish. A discrete model for releases of sterile mosquitoes with Beverton-Holt-type of survivability has been described by Li and Li [21].

Many researchers ([19], [20]) have paid their attention on different regions such as refuge region and predatory region and shown their impacts on prey-predator in these regions. To the best of our knowledge, for the first time, we consider the interaction among the generalist predator, predator and prey in reserve region. Here are two regions are considered, one is drainage system and other is form by lake, stream and river etc. where the drainage opens. The mosquito larvae generally grow in drainage system. To control their populations we have to cultivate gambezi in drainage system. But the drainage system is not a closed system, it opens at lake, river, stream etc. So gambezi comes from the drainage system. When they come out, we treat them as prey for the generalized predator like catfish. This is the new consideration in this paper, which is shown mathematically and analyzed the obtained results. In addition to the above, we choose the generalist predator for harvesting effort in this paper which is more realistic to analyze the whole system. Motivated by this idea, in this study, a mathematical model is designed and then analyzed.

The rest of the paper is sorted out as follows: mathematical model is presented in Section 2. In Sections 3 and 4, we describe the equilibria and their existence criteria and stability, respectively of the designed model. Optimal control is introduced to analyze the proposed model in Section 5. In order to verify the model, numerical simulation is presented in Section 6. Finally, conclusion with the future studies are described in Section 7.

\section{Mathematical structure}

To analyze the aforementioned situation practically, we choose here drainage basin in which one part is drainage system and the other part is formed by streams, rivers and lakes as shown in Figure 1 . The drainage system is opened in a certain place like as river and ocean. There are so many species which do not enter in drainage system like catfish (generalist predator) which consumes mosquito fish (Gambezi). Assuming that, at any time $t, x_{1}$ and $x_{2}$ represent the mosquito larva populations (preys) and gambezi populations (predators), respectively. Here, for mosquito fish, drainage system is a reserve region. Considering that, at any time $t$, $x_{3}$ and $x_{4}$ denote the gambezi population and catfish population in other region, respectively. Generally, the growth rate of a predator in the reserve region is different from other area. Due to this reason, different growth 


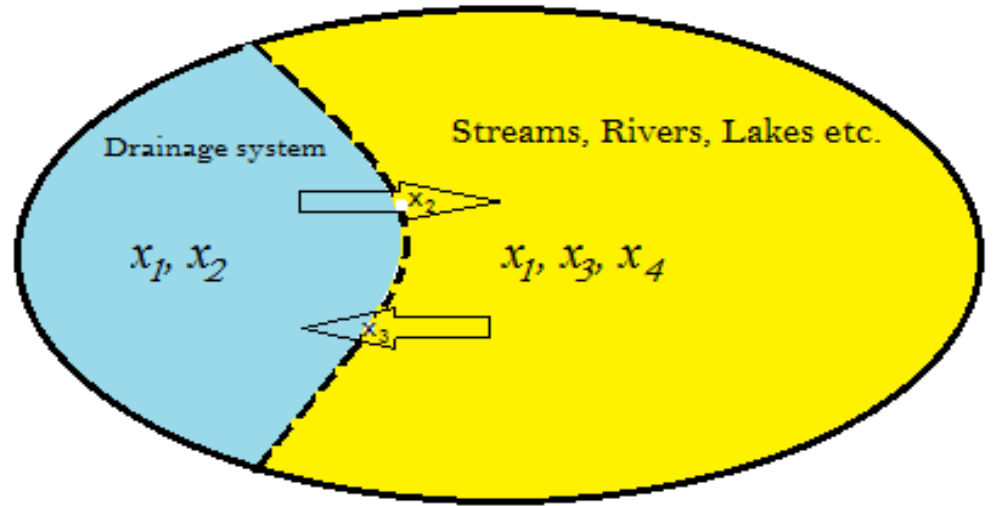

Figure 1: Pictorial representation of the model.

rates are considered for the predator in two different regions. Since the mosquito larva is considered as prey and gambezi as predator, the predator population consumes the prey population with Holling type-II functional response or Michaelis-Menten functional response which is chosen as $\frac{\chi_{1}}{a_{1}+\chi_{1}}$. The generalist predator population i.e., catfish consumes the gambezi, so predator population with Beddington-DeAngelis functional response is treated as $\beta \frac{x_{3}}{a+b x_{3}+m x_{4}}$, where $\beta, a, b$ and $m$ are positive integers.

In a reserved region, we select two regions which are connected to each other. So, migration as well as emigration should occur between the considered regions. Considering that, at any time $t, m_{1}$ is per unit emigration and $m_{2}$ denotes per unit migration of the predator population in the reserve region. The predator population can interact with prey population in all regions but the generalist predator population cannot interact with predator population in the reserve region.

The generalist predators i.e., catfishes are harvested for human needs. Without loss of generality, at any time $t$, the harvest rate is denoted by $h(t)$ and is defined by $h(t)=q E x_{4}$, where $q$ denotes as catchability co-efficient and $E$ represents the fishing effort for harvesting the generalist predator population. So, the system of equations is described as follows:

$$
\begin{aligned}
\frac{d x_{1}}{d t} & =r x_{1}\left(1-\frac{x_{1}}{K}\right)-\gamma \frac{x_{1} x_{2}}{a_{1}+x_{1}}-\gamma \frac{x_{1} x_{3}}{a_{1}+x_{1}}-d_{1} x_{1} \\
\frac{d x_{2}}{d t} & =s_{1} \frac{x_{1} x_{2}}{a_{1}+x_{1}}+m_{1} x_{3}-m_{2} x_{2} \\
\frac{d x_{3}}{d t} & =s_{2} \frac{x_{1} x_{3}}{a_{1}+x_{1}}-\beta \frac{x_{3} x_{4}}{a+b x_{3}+m x_{4}}-m_{1} x_{3}+m_{2} x_{2} \\
\frac{d x_{4}}{d t} & =\beta_{1} \frac{x_{3} x_{4}}{a+b x_{3}+m x_{4}}-d_{3} x_{4}-q E x_{4}
\end{aligned}
$$

with initial conditions $x_{1}(0) \geq 0, x_{2}(0) \geq 0, x_{3}(0) \geq 0$ and $x_{4}(0) \geq 0$. Since $x_{2}$ and $x_{3}$ are both predator populations, one is in the reserve region and another is in other region, respectively. To simplicity of the calculation and the predators $x_{2}$ and $x_{3}$ are the same species, the term of functional response of predator on prey in reserve region and other region is treated as same. So, the system of equations (1) is described as follows:

$$
\begin{aligned}
\frac{d x_{1}}{d t} & =r x_{1}\left(1-\frac{x_{1}}{K}\right)-\alpha \frac{x_{1} x_{2}}{a_{1}+x_{1}}-d_{1} x_{1} \\
\frac{d x_{2}}{d t} & =s_{1} \frac{x_{1} x_{2}}{a_{1}+x_{1}}+m_{1} x_{3}-m_{2} x_{2} \\
\frac{d x_{3}}{d t} & =s_{2} \frac{x_{1} x_{3}}{a_{1}+x_{1}}-\beta \frac{x_{3} x_{4}}{a+b x_{3}+m x_{4}}-m_{1} x_{3}+m_{2} x_{2} \\
\frac{d x_{4}}{d t} & =\beta_{1} \frac{x_{3} x_{4}}{a+b x_{3}+m x_{4}}-d_{3} x_{4}-q E x_{4}
\end{aligned}
$$


with initial conditions $x_{1}(0) \geq 0, x_{2}(0) \geq 0, x_{3}(0) \geq 0, x_{4}(0) \geq 0$ and $\alpha=2 \gamma$. Description of the parameters which are mentioned in mathematical structure of the model is given in Table 1.

Table 1: Description of Parameters.

\begin{tabular}{ll}
\hline Parameter & Description of the parameters \\
\hline$x_{1}$ & Population of prey at time $t$ \\
$x_{2}$ & Population of predator in reserve region at time $t$ \\
$x_{3}$ & Population of predator in other region at time $t$ \\
$x_{4}$ & Population of generalist predator at time $t$ \\
$r$ & Intrinsic growth rate of prey \\
$K$ & Environmental carrying capacity of the prey \\
$\gamma$ & Maximal predator per capita consumption rate \\
$a_{1}$ & Half saturation constants \\
$d_{1}$ & Natural death rate of prey \\
$d_{3}$ & Natural death rate of generalist predator \\
$s_{1}, s_{2}$ & Growth rate of Predator in reserve region and other region, respectively \\
$\beta$ & Generalist predator's consumption rate on predator \\
$\beta_{1}$ & Growth rate of Generalist predator \\
$m_{1}$ & Per unit emigration of the predator population in reserve region \\
$m_{2}$ & Per unit migration of the predator population in reserve region \\
$q$ & Catchability co-efficient \\
$E$ & Fishing effort for harvesting the generalist predator population \\
\hline
\end{tabular}

\section{Equilibria and their existence criteria}

To analyze the system (2) at its interior equilibrium point we first try to find all possible non-negative equilibria of the system, though we particularly, interest about the analysis around the interior equilibrium point $P\left(\bar{x}_{1}, \bar{x}_{2}, \bar{x}_{3}, \bar{x}_{4}\right)$ to the system (2) for its natural importance.

(i) The trivial equilibrium point $(0,0,0,0)$.

(ii) The prey and generalist predator free equilibrium point $\left(0, \hat{x}_{2}, \hat{x}_{3}, 0\right)$ where $\hat{x}_{2}=\frac{a_{1}}{\alpha}\left(r-d_{1}\right)$ and $\hat{x}_{3}=$ $\frac{m_{2} a_{1}}{m_{1} \alpha}\left(r-d_{1}\right)$ exist if growth rate of prey is greater than death rate of prey.

(iii) The equilibrium point $\left(x_{1}^{\star}, x_{2}^{\star}, 0,0\right)$, where

$$
\begin{aligned}
x_{1}^{*}= & \frac{m_{2} a_{1}}{s_{1}-m_{2}}, \\
x_{2}^{*}= & \frac{a+x_{1}^{*}}{\alpha}\left\{r\left(1-\frac{x_{1}^{*}}{K}\right)-d_{1}\right\},
\end{aligned}
$$

So, the sufficient conditions for the system at the equilibrium point $\left(x_{1}^{\star}, x_{2}^{\star}, 0,0\right)$ are $s_{1}>m_{2}$ and $r\left(1-\frac{x_{1}^{*}}{K}\right)>$ $d_{1}$. i.e., logistic growth of predator in reserve region is greater than the migration of predator and growth rate of prey is greater than death rate of prey.

(iv) The steady state of system of equations (2) is calculated by solving the equations. Let the interior equilibrium point be denoted by $P\left(\bar{x}_{1}, \bar{x}_{2}, \bar{x}_{3}, \bar{x}_{4}\right)$ where $\bar{x}_{1}, \bar{x}_{2}, \bar{x}_{3}$ and $\bar{x}_{4}$ are the positive roots of the equations $\dot{x_{1}}=\dot{x_{2}}=\dot{x_{3}}=\dot{x_{4}}=0$, where $\dot{x}_{i}=\frac{d x_{i}}{d t}, i=1,2,3$ and 4 . 
On solving the equations we have the following:

$$
\begin{aligned}
& \bar{x}_{4}=\frac{1}{m}\left\{\left(\frac{\beta_{1}}{d_{3}+q E}-b\right) \bar{x}_{3}-a\right\}, \\
& \bar{x}_{3}=\frac{1}{m_{1}}\left\{m_{2} \bar{x}_{2}-s_{1} \frac{\bar{x}_{1} \bar{x}_{2}}{a_{1}+\bar{x}_{1}}\right\}, \\
& \bar{x}_{2}=\frac{a_{1}+\bar{x}_{1}}{\alpha}\left\{r\left(1-\frac{\bar{x}_{1}}{K}\right)-d_{1}\right\},
\end{aligned}
$$

and $\bar{x}_{1}$ is the positive root of the following equation

$$
B_{5} x_{1}^{5}+B_{4} x_{1}^{4}+B_{3} x_{1}^{3}+B_{2} x_{1}^{2}+B_{1} x_{1}-B_{0}=0,
$$

where

$$
\begin{array}{ll}
B_{0}= & \beta a_{1} C_{8}\left(C_{1} C_{8}+C_{2}\right), \\
B_{1}= & C_{8}^{2}\left\{s_{2}\left(b+C_{1} m\right)-\beta C_{1}\right\}+C_{8}\left\{s_{2}\left(a+C_{2} m\right)+C_{5} s_{1}\left(b+C_{1} m\right)-\beta C_{2}\right\} \\
& -\beta a_{1} C_{7}\left(2 C_{1} C_{8}+C_{2}\right)+C_{5} s_{1}\left(a+m C_{2}\right), \\
B_{2}= & -2 \beta a_{1} C_{1} C_{7} C_{8}\left(C_{7}^{2}+2 C_{6} C_{8}\right)+2 C_{7} C_{8}\left\{s_{2}\left(b+C_{1} m\right)-\beta C_{1}\right\}-\beta a_{1} C_{2} C_{6} \\
& +C_{7}\left\{s_{2}\left(a+C_{2} m\right)+C_{5} s_{1}\left(b+C_{1} m\right)-\beta C_{2}\right\}+s_{1} C_{4}\left(a+m C_{2}\right), \\
B_{3}=\quad & \left(C_{7}^{2}+2 C_{6} C_{8}\right)\left\{s_{2}\left(b+C_{1} m\right)-\beta C_{1}\right\}+C_{6}\left\{s_{2}\left(a+C_{2} m\right)+C_{5} s_{1}\left(b+C_{1} m\right)-\beta C_{2}\right\} \\
& +C_{7} C_{4} s_{1}\left(b+C_{1} m\right)+s_{1} C_{3}\left(a+m C_{2}\right)-2 \beta a_{1} C_{1} C_{6} C_{7}, \\
B_{4}=\quad & 2 C_{6} C_{7}\left\{s_{2}\left(b+C_{1} m\right)-\beta C_{1}\right\}+C_{6} C_{4} s_{1}\left(b+C_{1} m\right)+C_{7} C_{3} s_{1}\left(b+C_{1} m\right)-\beta a_{1} C_{1} C_{6}^{2}, \\
B_{5}= & C_{6}^{2}\left\{s_{2}\left(b+C_{1} m\right)-\beta C_{1}\right\}+C_{6} C_{3} s_{1}\left(b+C_{1} m\right),
\end{array}
$$

and

$$
\begin{array}{llrl}
C_{1}= & \frac{1}{m}\left(\frac{\beta_{1}}{d_{3}+q E}-b\right), & C_{5}=\frac{a_{1}}{\alpha}\left(r-d_{1}\right), \\
C_{2}=-\frac{a}{m}, & C_{6}=\frac{r}{m_{1} \alpha K}\left(s_{1}-m_{2}\right), \\
C_{3}=-\frac{r}{\alpha K}, & C_{7}=\frac{1}{\alpha m_{1}}\left\{\left(m_{2}-s_{1}\right)\left(r-d_{1}\right)-a_{1} m_{2} \frac{r}{K}\right\}, \\
C_{4}= & \frac{1}{\alpha}\left(r-d_{1}-a_{1} \frac{r}{K}\right), & C_{8}=\frac{a_{1} m_{2}}{\alpha m_{1}}\left(r-d_{1}\right) .
\end{array}
$$

So, the sufficient conditions for the system with a positive interior equilibrium point are $r>d_{1}, \beta_{1}>b\left(d_{3}+q E\right)$ and for all $B_{i}(i=0,1,2,3,4,5)$ are positive. i.e., condition for a positive equilibrium point, the intrinsic growth rate of prey is greater than the natural death rate of prey, and growth rate of generalist predator is greater than the sum of natural death rate of generalist predator and harvesting rate of generalist predator.

\section{Local stability}

The stability criterion of the system is analyzed at the interior equilibrium point. The Jacobian matrix of the system (2) at the interior equilibrium point $P$ is denoted by $V$ and is defined as follows:

$$
V=\left(\begin{array}{cccc}
-\frac{r}{K} \bar{x}_{1}+\alpha \frac{\bar{x}_{1} \bar{x}_{2}}{\left(a_{1}+\bar{x}_{1}\right)^{2}} & -\alpha \frac{\bar{x}_{1}}{a_{1}+\bar{x}_{1}} & 0 & 0 \\
\frac{a_{1} s_{1} \bar{x}_{2}}{\left(a_{1}+\bar{x}_{1}\right)^{2}} & \frac{s_{1} \bar{x}_{1}}{a_{1}+\bar{x}_{1}}-m_{2} & m_{1} & 0 \\
\frac{a_{1} s_{2} \bar{x}_{3}}{\left(a_{1}+\bar{x}_{1}\right)^{2}} & m_{2} & \frac{s_{2} \bar{x}_{1}}{a_{1}+\bar{x}_{1}}-m_{1}-\beta \bar{x}_{4} \frac{a+m \bar{x}_{4}}{\left(a+b \bar{x}_{3}+m \bar{x}_{4}\right)^{2}} & -\frac{\beta \bar{x}_{3}\left(a+b \bar{x}_{3}\right)}{\left(a+b \bar{x}_{3}+m \bar{x}_{4}\right)^{2}} \\
0 & 0 & \frac{\beta_{1} \bar{x}_{4}\left(a+m \bar{x}_{4}\right)}{\left(a+b \bar{x}_{3}+m \bar{x}_{4}\right)^{2}} & -\frac{m \beta_{1} \bar{x}_{3} \bar{x}_{4}}{\left(a+b \bar{x}_{3}+m \bar{x}_{4}\right)^{2}}
\end{array}\right) .
$$

Now, the characteristic equation of system (2) around its interior equilibrium is $\operatorname{det}(V-\lambda I)=0$, i.e., $\lambda^{4}+h_{1} \lambda^{3}+h_{2} \lambda^{2}+h_{3} \lambda+h_{4}=0$. 
where $I$ represents an identity matrix of order 4 and

$$
\begin{aligned}
& h_{1}=\frac{r}{K} \bar{x}_{1}-\alpha \frac{\bar{x}_{1} \bar{x}_{2}}{\left(a_{1}+\bar{x}_{1}\right)^{2}}-\frac{s_{1} \bar{x}_{1}+s_{2} \bar{x}_{1}}{a_{1}+\bar{x}_{1}}+m_{2}+m_{1}+\bar{x}_{4} \frac{\left\{\beta\left(a+m \bar{x}_{4}\right)+\beta_{1} m \bar{x}_{3}\right\}}{\left(a+b \bar{x}_{3}+m \bar{x}_{4}\right)^{2}}, \\
& h_{2}=r_{1} r_{2}+r_{1} r_{3}+r_{1} r_{4}+r_{2} r_{3}+r_{2} r_{4}+r_{3} r_{4}-m_{1} m_{2}+\frac{\beta \beta_{1} \bar{x}_{3} \bar{x}_{4}\left(a+b \bar{x}_{3}\right)\left(a+m \bar{x}_{4}\right)}{\left(a+b \bar{x}_{3}+m \bar{x}_{4}\right)^{4}} \\
& +\frac{\alpha a_{1} s_{1} \bar{x}_{1} \bar{x}_{2}}{\left(a_{1}+\bar{x}_{1}\right)^{3}} \\
& h_{3}=-\left(r_{1} r_{2} r_{3}+r_{1} r_{3} r_{4}+r_{1} r_{2} r_{4}+r_{2} r_{3} r_{4}\right)+m_{1} m_{2}\left(r_{1}+r_{4}\right) \\
& -\frac{\beta \beta_{1} \bar{x}_{3} \bar{x}_{4}\left(a+b \bar{x}_{3}\right)\left(a+m \bar{x}_{4}\right)}{\left(a+b \bar{x}_{3}+m \bar{x}_{4}\right)^{4}}\left(r_{1}+r_{2}\right)-\frac{\alpha a_{1} s_{1} \bar{x}_{1} \bar{x}_{2}}{\left(a_{1}+\bar{x}_{1}\right)^{3}}\left(r_{3}+r_{4}\right)+m_{1} \alpha a_{1} s_{2} \frac{\bar{x}_{1} \bar{x}_{3}}{\left(a_{1}+\bar{x}_{1}\right)^{3}}, \\
& h_{4}=\left\{r_{1} r_{2}+\frac{\alpha a_{1} s_{1} \bar{x}_{1} \bar{x}_{2}}{\left(a_{1}+\bar{x}_{1}\right)^{3}}\right\}\left\{\frac{\beta \beta_{1} \bar{x}_{3} \bar{x}_{4}\left(a+b \bar{x}_{3}\right)\left(a+m \bar{x}_{4}\right)}{\left(a+b \bar{x}_{3}+m \bar{x}_{4}\right)^{4}}+r_{3} r_{4}\right\}-m_{1} m_{2} r_{1} r_{4} \\
& -\alpha m_{1} a_{1} s_{2} \frac{\bar{x}_{1} \bar{x}_{3} r_{4}}{\left(a_{1}+\bar{x}_{1}\right)^{3}}, \\
& r_{1}=-\frac{r}{K} \bar{x}_{1}+\alpha \frac{\bar{x}_{1} \bar{x}_{2}}{\left(a_{1}+\bar{x}_{1}\right)^{2}}, \quad r_{2}=\frac{s_{1} \bar{x}_{1}}{a_{1}+\bar{x}_{1}}-m_{2}, \\
& r_{3}=\frac{s_{2} \bar{x}_{1}}{a_{1}+\bar{x}_{1}}-m_{1}-\beta \bar{x}_{4} \frac{a+m \bar{x}_{4}}{\left(a+b \bar{x}_{3}+m \bar{x}_{4}\right)^{2}}, \quad r_{4}=-\frac{m \beta_{1} \bar{x}_{3} \bar{x}_{4}}{\left(a+b \bar{x}_{3}+m \bar{x}_{4}\right)^{2}} \text {. }
\end{aligned}
$$

Denoting here $h_{1}=\frac{c_{1}}{K}-c_{2}, h_{2}=\frac{c_{3}}{K}-c_{4}, h_{3}=\frac{c_{5}}{K}-c_{6}, h_{4}=\frac{c_{7}}{K}-c_{8}$.

Where

$$
\begin{aligned}
& c_{1}=r \bar{x}_{1} \text {, } \\
& c_{2}=\alpha \frac{\bar{x}_{1} \bar{x}_{2}}{\left(a_{1}+\bar{x}_{1}\right)^{2}}+\frac{\left(s_{1}+s_{2}\right) \bar{x}_{1}}{a_{1}+\bar{x}_{1}}-m_{2}-m_{1}-\bar{x}_{4} \frac{\beta\left(a+m \bar{x}_{4}\right)+m \beta_{1} \bar{x}_{3}}{\left(a+b \bar{x}_{3}+m \bar{x}_{4}\right)^{2}}, \\
& c_{3}=-r \bar{x}_{1}\left\{\frac{\left(s_{1}+s_{2}\right) \bar{x}_{1}}{a_{1}+\bar{x}_{1}}-m_{2}-m_{1}-\bar{x}_{4} \frac{\beta\left(a+m \bar{x}_{4}\right)+m \beta_{1} \bar{x}_{3}}{\left(a+b \bar{x}_{3}+m \bar{x}_{4}\right)^{2}}\right\}, \\
& c_{4}=\alpha \frac{\bar{x}_{1} \bar{x}_{2}}{\left(a_{1}+\bar{x}_{1}\right)^{2}}\left\{\frac{\left(s_{1}+s_{2}\right) \bar{x}_{1}}{a_{1}+\bar{x}_{1}}-m_{2}-m_{1}-\bar{x}_{4} \frac{\beta\left(a+m \bar{x}_{4}\right)+m \beta_{1} \bar{x}_{3}}{\left(a+b \bar{x}_{3}+m \bar{x}_{4}\right)^{2}}\right\} \\
& +r_{2} r_{3}+r_{2} r_{4}+r_{3} r_{4}-m_{1} m_{2}+\frac{\beta \beta_{1} \bar{x}_{3} \bar{x}_{4}\left(a+b \bar{x}_{3}\right)\left(a+m \bar{x}_{4}\right)}{\left(a+b \bar{x}_{3}+m \bar{x}_{4}\right)^{4}}+\frac{\alpha a_{1} s_{1} \bar{x}_{1} \bar{x}_{2}}{\left(a_{1}+\bar{x}_{1}\right)^{3}}, \\
& c_{5}=r \bar{x}_{1}\left\{\left(r_{2} r_{3}+r_{3} r_{4}+r_{2} r_{4}\right)-m_{1} m_{2}-\frac{\beta \beta_{1} \bar{x}_{3} \bar{x}_{4}\left(a+b \bar{x}_{3}\right)\left(a+m \bar{x}_{4}\right)}{\left(a+b \bar{x}_{3}+m \bar{x}_{4}\right)^{4}}\right\} \\
& c_{6}=\alpha \frac{\bar{x}_{1} \bar{x}_{2}}{\left(a_{1}+\bar{x}_{1}\right)^{2}}\left(r_{2} r_{3}+r_{3} r_{4}+r_{2} r_{4}\right)-m_{1} m_{2}\left\{\alpha \frac{\bar{x}_{1} \bar{x}_{2}}{\left(a_{1}+\bar{x}_{1}\right)^{2}}+r_{4}\right\}-m_{1} \alpha a_{1} s_{2} \frac{\bar{x}_{1} \bar{x}_{3}}{\left(a_{1}+\bar{x}_{1}\right)^{3}} \\
& +\frac{\beta \beta_{1} \bar{x}_{3} \bar{x}_{4}\left(a+b \bar{x}_{3}\right)\left(a+m \bar{x}_{4}\right)}{\left(a+b \bar{x}_{3}+m \bar{x}_{4}\right)^{4}}\left(\alpha \frac{\bar{x}_{1} \bar{x}_{2}}{\left(a_{1}+\bar{x}_{1}\right)^{2}}+r_{2}\right)+\frac{\alpha a_{1} s_{1} \bar{x}_{1} \bar{x}_{2}}{\left(a_{1}+\bar{x}_{1}\right)^{3}}\left(r_{3}+r_{4}\right)+r_{2} r_{3} r_{4}, \\
& c_{7}=r \bar{x}_{1}\left\{m_{2}-\frac{s_{1} \bar{x}_{1}}{a_{1}+\bar{x}_{1}}\right\}\left\{\frac{\beta \beta_{1} \bar{x}_{3} \bar{x}_{4}\left(a+b \bar{x}_{3}\right)\left(a+m \bar{x}_{4}\right)}{\left(a+b \bar{x}_{3}+m \bar{x}_{4}\right)^{4}}+r_{3} r_{4}\right\}+m_{1} m_{2} r r_{4} \bar{x}_{1} \text {, } \\
& c_{8}=\left\{\alpha \frac{\bar{x}_{1} \bar{x}_{2}}{\left(a_{1}+\bar{x}_{1}\right)^{2}}\left(m_{2}-\frac{s_{1} \bar{x}_{1}}{a_{1}+\bar{x}_{1}}\right)-\frac{\alpha a_{1} s_{1} \bar{x}_{1} \bar{x}_{2}}{\left(a_{1}+\bar{x}_{1}\right)^{3}}\right\}\left\{\frac{\beta \beta_{1} \bar{x}_{3} \bar{x}_{4}\left(a+b \bar{x}_{3}\right)\left(a+m \bar{x}_{4}\right)}{\left(a+b \bar{x}_{3}+m \bar{x}_{4}\right)^{4}}+r_{3} r_{4}\right\} \\
& +m_{1} m_{2} r_{4} \alpha \frac{\bar{x}_{1} \bar{x}_{2}}{\left(a_{1}+\bar{x}_{1}\right)^{2}}+\alpha m_{1} a_{1} s_{2} \frac{\bar{x}_{1} \bar{x}_{3} r_{4}}{\left(a_{1}+\bar{x}_{1}\right)^{3}},
\end{aligned}
$$

If we choose $\frac{1}{K}=K_{1}$, then, we have $h_{2} h_{3}-h_{1} h_{4}=Q_{1}+Q_{2} K_{1}+Q_{3} K_{1}^{2}=\varphi\left(K_{1}\right)$ (say), where $Q_{1}=c_{4} c_{6}-c_{2} c_{8}$, $Q_{2}=c_{2} c_{7}+c_{1} c_{8}-c_{5} c_{4}-c_{3} c_{6}, Q_{3}=c_{3} c_{5}-c_{1} c_{7}$ and det $\left(\begin{array}{ccc}h_{3} & h_{4} & 0 \\ h_{1} & h_{2} & h_{3} \\ 0 & 1 & h_{1}\end{array}\right)=Q_{4}+Q_{5} K_{1}+Q_{6} K_{1}^{2}+Q_{7} K_{1}^{3}=\psi\left(K_{1}\right)$ (say), where $Q_{4}=c_{2}^{2} c_{8}-c_{6}^{2}-c_{2} c_{4} c_{6}, Q_{5}=c_{2} c_{4} c_{5}+c_{2} c_{3} c_{6}+c_{1} c_{4} c_{6}-c_{2}^{2} c_{7}-2 c_{1} c_{2} c_{8}+2 c_{5} c_{6}, Q_{6}=$ $c_{1}^{2} c_{8}+2 c_{1} c_{2} c_{7}-c_{2} c_{3} c_{5}-c_{1} c_{4} c_{5}-c_{1} c_{3} c_{6}-c_{5}^{2}, Q_{7}=c_{1} c_{3} c_{5}-c_{1}^{2} c_{7}$.

Here all $Q_{j}(j=1,2, \ldots, 7)$ are functions of $K_{1}$, as the interior equilibrium point depends on $K_{1}$. But for a 
known parameter set, it is possible to find all the values of $Q_{j}$ in terms of $K_{1}$. In that case, we assume that $\bar{K}_{1}$ is the common positive root of $\varphi\left(K_{1}\right)=0$ and $\psi\left(K_{1}\right)=0$. Now using Routh-Hurwitz criteria around at the interior equilibrium point, we can now state and prove two theorems for analyzing the local stability of the system (2).

Theorem 1: Considering that all $Q_{j}(j=1,2, \ldots, 7), h_{2} h_{3}-h_{1} h_{4}$ and $\psi\left(K_{1}\right)$ are positive. Then at the equilibrium point $P\left(\bar{x}_{1}, \bar{x}_{2}, \bar{x}_{3}, \bar{x}_{4}\right)$ of the system (2) is locally asymptotically stable, i.e., after a resilience the system comes to the equilibrium state.

Proof: Using Routh-Hurwitz criteria, the conclusion becomes all eigenvalues of the system (2) around its interior equilibrium point $P\left(\bar{x}_{1}, \bar{x}_{2}, \bar{x}_{3}, \bar{x}_{4}\right)$ have negative real parts. Consequently the system will be locally asymptotically stable. This completes the proof of the theorem.

Lemma 1: In Theorem 1, it is proved that for a known parameter set, we find all $Q_{j}$ in terms of $K_{1}$. In this case if $\overline{K_{1}}$ is the only common positive root. Then for $K_{1}>\bar{K}_{1}, h_{2} h_{3}-h_{1} h_{4}$ and $\psi\left(K_{1}\right)$ are positive. If $h_{2} h_{3}-h_{1} h_{4}$ and $\psi\left(K_{1}\right)$ are negative then the system (2) will be unstable around $\bar{P}\left(\bar{x}_{1}, \bar{x}_{2}, \bar{x}_{3}, \bar{x}_{4}\right)$.

Also if $\psi\left(K_{1}\right)=0$, then the system (2) undergoes through a bifurcation.

Again $K_{1}=\frac{1}{K}$, thus we have, for $K<\bar{K}, h_{2} h_{3}-h_{1} h_{4}$ and $\psi\left(K_{1}\right)$ are positive. If $h_{2} h_{3}-h_{1} h_{4}$ and $\psi\left(K_{1}\right)$ are negative then the system (2) must be unstable around $\bar{P}\left(\bar{x}_{1}, \bar{x}_{2}, \bar{x}_{3}, \bar{x}_{4}\right)$. Also if $\psi\left(K_{1}\right)=0$, then the system (2) undergoes through a bifurcation.

After a certain time by cultivating gambezi in a drainage system, mosquito larvae will be controlled automatically for $K_{1}>\bar{K}_{1}$.

Theorem 2: System (2) undergoes through Hopf bifurcation around $\bar{P}\left(\bar{x}_{1}, \bar{x}_{2}, \bar{x}_{3}, \bar{x}_{4}\right)$ for $K_{1}=\bar{K}_{1}$.

Proof: For $K_{1}=\bar{K}_{1}$, we have $\psi\left(K_{1}\right)=0$ and then the eigenvalues of the system at $\bar{P}\left(\bar{x}_{1}, \bar{x}_{2}, \bar{x}_{3}, \bar{x}_{4}\right)$ which can be expressed as $\lambda_{1,2}=\phi_{1}\left(K_{1}\right) \pm i \phi_{2}\left(K_{1}\right)$ and $\lambda_{3,4}=\phi_{3}\left(K_{1}\right) \pm i \phi_{4}\left(K_{1}\right)$. Now it is easy to show that $\frac{d \phi}{d K_{1}}$ is non-zero at the point $K_{1}=\overline{K_{1}}$. Also we see $\phi\left(\overline{K_{1}}\right)=0$ and $\left\{\frac{\partial}{\partial K_{1}} \operatorname{tr}\left(\left.V\right|_{\bar{P}}\right)\right\}_{K_{1}=\overline{K_{1}}}=-r \overline{x_{1}}<0$. Hence, using the given conditions in Venkatsubramanian et al. [22], it is clear to show that our system (2) undergoes a Hopf bifurcation at its interior equilibrium point for the critical value of $K_{1}$, i.e., for $K_{1}=\bar{K}_{1}$. This completes the proof of the theorem.

Now we present the condition that the system will be globally stable at the equilibrium point $\bar{P}\left(\bar{x}_{1}, \bar{x}_{2}, \bar{x}_{3}, \bar{x}_{4}\right)$.

Theorem 3: The sufficient conditions for the system (2) is globally asymptotically stable at its interior equilibrium $\bar{P}\left(\bar{x}_{1}, \bar{x}_{2}, \bar{x}_{3}, \bar{x}_{4}\right)$ are $\frac{r}{K}>\frac{\alpha x_{2}}{\left(a_{1}+\bar{x}_{1}\right)\left(a_{1}+x_{1}\right)}+\frac{a_{1} s_{2}}{2\left(a_{1}+x_{1}\right)\left(a_{1}+\bar{x}_{1}\right)}, \quad \frac{m_{1} x_{3} \alpha\left(a_{1}+x_{1}\right)}{x_{2} \bar{x}_{2} a_{1} s_{1}}>\frac{1}{2}\left(\frac{m_{1} \alpha\left(a_{1}+x_{1}\right)}{a_{1} s_{1} \bar{x}_{2}}+\frac{m_{2}}{\bar{x}_{3}}\right)$ and $\frac{m_{2} x_{2}}{\bar{x}_{3} \bar{x}_{3}}+b \beta \frac{\bar{x}_{4}}{\left(a+b x_{3}+m x_{4}\right)\left(a+b \bar{x}_{3}+m \bar{x}_{4}\right)}>\frac{1}{2}\left(\frac{m_{1} \alpha\left(a_{1}+x_{1}\right)}{a_{1} s_{1} \bar{x}_{2}}+\frac{m_{2}}{\bar{x}_{3}}\right)+\frac{a_{1} s_{2}}{2\left(a_{1}+x_{1}\right)\left(a_{1}+\bar{x}_{1}\right)}$.

Proof: Let us choose a Lyapunov function defined as follows:

$W\left(x_{1}, x_{2}, x_{3}, x_{4}\right)=\int_{\bar{x}_{1}}^{x_{1}} \frac{x_{1}-\bar{x}_{1}}{x_{1}} d x_{1}+p_{1} \int_{\bar{x}_{2}}^{x_{2}} \frac{x_{2}-\bar{x}_{2}}{x_{2}} d x_{2}+p_{2} \int_{\bar{x}_{3}}^{x_{3}} \frac{x_{3}-\overline{x_{3}}}{x_{3}} d x_{3}+p_{3} \int_{\bar{x}_{4}}^{x_{4}} \frac{x_{4}-\bar{x}_{4}}{x_{4}} d x_{4}$ where $p_{i}(i=1,2,3)$ are suitable positive constants to be determined in the following subsequent steps. Time derivative of the equation along the solutions of the system (2) is given by

$$
\begin{aligned}
\frac{d W}{d t}=\quad & \frac{x_{1}-\overline{x_{1}}}{x_{1}} \frac{d x_{1}}{d t}+p_{1} \frac{x_{2}-\overline{x_{2}}}{x_{2}} \frac{d x_{2}}{d t}+p_{2} \frac{x_{3}-\overline{x_{3}}}{x_{3}} \frac{d x_{3}}{d t}+p_{3} \frac{x_{4}-\overline{x_{4}}}{x_{4}} \frac{d x_{4}}{d t} \\
= & \left(x_{1}-\bar{x}_{1}\right)\left\{-\frac{r}{K}\left(x_{1}-\bar{x}_{1}\right)+\alpha\left(\frac{x_{2}}{a_{1}+x_{1}}-\frac{\bar{x}_{2}}{a_{1}+\bar{x}_{1}}\right)\right\} \\
& +p_{1}\left(x_{2}-\bar{x}_{2}\right)\left\{s_{1}\left(\frac{x_{1}}{a_{1}+x_{1}}-\frac{\bar{x}_{1}}{a_{1}+\bar{x}_{1}}\right)+m_{1}\left(\frac{x_{3}}{x_{2}}-\frac{\bar{x}_{3}}{\bar{x}_{2}}\right)\right\}
\end{aligned}
$$




$$
\begin{aligned}
& +p_{2}\left(x_{3}-\bar{x}_{3}\right)\left\{s_{2}\left(\frac{x_{1}}{a_{1}+x_{1}}-\frac{\bar{x}_{1}}{a_{1}+\bar{x}_{1}}\right)+m_{2}\left(\frac{x_{2}}{x_{3}}-\frac{\bar{x}_{2}}{\bar{x}_{3}}\right)\right. \\
& \left.-\beta\left(\frac{x_{4}}{\left(a+b x_{3}+m x_{4}\right)}-\frac{\bar{x}_{4}}{\left(a+b \bar{x}_{3}+m \bar{x}_{4}\right)}\right)\right\} \\
& +p_{3}\left(x_{4}-\overline{x_{4}}\right) \beta_{1}\left(\frac{x_{3}}{\left(a+b x_{3}+m x_{4}\right)}-\frac{\bar{x}_{3}}{\left(a+b \bar{x}_{3}+m \bar{x}_{4}\right)}\right) \\
& =-\left(\frac{r}{K}-\frac{\alpha x_{2}}{\left(a_{1}+\bar{x}_{1}\right)\left(a_{1}+x_{1}\right)}\right)\left(x_{1}-\bar{x}_{1}\right)^{2}-p_{1} \frac{m_{1} x_{3}}{x_{2} \bar{x}_{2}}\left(x_{2}-\bar{x}_{2}\right)^{2} \\
& -p_{2}\left(\frac{m_{2} x_{2}}{x_{3} \bar{x}_{3}}+b \beta \frac{\bar{x}_{4}}{\left(a+b x_{3}+m x_{4}\right)\left(a+b \bar{x}_{3}+m \bar{x}_{4}\right)}\right)\left(x_{3}-\bar{x}_{3}\right)^{2} \\
& -p_{3} \frac{m \beta_{1} \bar{x}_{3}}{\left(a+b x_{3}+m x_{4}\right)\left(a+b \bar{x}_{3}+m \bar{x}_{4}\right)}\left(x_{4}-\bar{x}_{4}\right)^{2}+\left(\frac{p_{1} a_{1} s_{1}}{\left(a_{1}+x_{1}\right)\left(a_{1}+\bar{x}_{1}\right)}\right. \\
& \left.-\frac{\alpha}{a_{1}+\bar{x}_{1}}\right)\left(x_{1}-\bar{x}_{1}\right)\left(x_{2}-\bar{x}_{2}\right)+\left(\frac{p_{1} m_{1}}{\bar{x}_{2}}+\frac{p_{2} m_{2}}{\bar{x}_{3}}\right)\left(x_{2}-\bar{x}_{2}\right)\left(x_{3}-\bar{x}_{3}\right) \\
& +\frac{p_{3} \beta_{1}\left(a+m \bar{x}_{4}\right)-p_{2} \beta\left(a+b \bar{x}_{3}\right)}{\left(a+b x_{3}+m x_{4}\right)\left(a+b \bar{x}_{3}+m \bar{x}_{4}\right)}\left(x_{3}-\bar{x}_{3}\right)\left(x_{4}-\bar{x}_{4}\right) \\
& +\frac{p_{2} a_{1} s_{2}}{\left(a_{1}+x_{1}\right)\left(a_{1}+\bar{x}_{1}\right)}\left(x_{1}-\bar{x}_{1}\right)\left(x_{3}-\bar{x}_{3}\right)
\end{aligned}
$$

Now let us consider, $p_{1}=\frac{\alpha\left(a_{1}+x_{1}\right)}{a_{1} s_{1}}, p_{2}=1, \quad p_{3}=\frac{\beta\left(a+b \bar{x}_{3}\right)}{\beta_{1}\left(a+m \bar{x}_{4}\right)}$

Then the above expression reduces as follows:

$$
\begin{aligned}
& \frac{d W}{d t}=-\left(\frac{r}{K}-\frac{\alpha x_{2}}{\left(a_{1}+\bar{x}_{1}\right)\left(a_{1}+x_{1}\right)}\right)\left(x_{1}-\bar{x}_{1}\right)^{2}-\frac{\alpha\left(a_{1}+x_{1}\right)}{a_{1} s_{1}} \times \frac{m_{1} x_{3}}{x_{2} \bar{x}_{2}}\left(x_{2}-\bar{x}_{2}\right)^{2} \\
& -\left(\frac{m_{2} x_{2}}{x_{3} \bar{x}_{3}}+b \beta \frac{\bar{x}_{4}}{\left(a+b x_{3}+m x_{4}\right)\left(a+b \bar{x}_{3}+m \bar{x}_{4}\right)}\right)\left(x_{3}-\bar{x}_{3}\right)^{2} \\
& -\frac{\beta\left(a+b \bar{x}_{3}\right)}{\left(a+m \bar{x}_{4}\right)} \times \frac{m \bar{x}_{3}}{\left(a+b x_{3}+m x_{4}\right)\left(a+b \bar{x}_{3}+m \bar{x}_{4}\right)}\left(x_{4}-\bar{x}_{4}\right)^{2} \\
& +\left(\frac{m_{1} \alpha\left(a_{1}+x_{1}\right)}{a_{1} s_{1} \bar{x}_{2}}+\frac{m_{2}}{\bar{x}_{3}}\right)\left(x_{2}-\bar{x}_{2}\right)\left(x_{3}-\bar{x}_{3}\right) \\
& +\frac{a_{1} s_{2}}{\left(a_{1}+x_{1}\right)\left(a_{1}+\bar{x}_{1}\right)}\left(x_{1}-\bar{x}_{1}\right)\left(x_{3}-\bar{x}_{3}\right) \\
& \frac{d W}{d t}=\quad-\left[\sqrt{\frac{1}{2}\left(\frac{m_{1} \alpha\left(a_{1}+x_{1}\right)}{a_{1} s_{1} \bar{x}_{2}}+\frac{m_{2}}{\bar{x}_{3}}\right)}\left\{\left(x_{2}-\bar{x}_{2}\right)-\left(x_{3}-\bar{x}_{3}\right)\right\}\right]^{2} \\
& -\left[\sqrt{\frac{a_{1} s_{2}}{2\left(a_{1}+x_{1}\right)\left(a_{1}+\bar{x}_{1}\right)}}\left\{\left(x_{1}-\bar{x}_{1}\right)-\left(x_{3}-\bar{x}_{3}\right)\right\}\right]^{2} \\
& -\left(\frac{r}{K}-\frac{\alpha x_{2}}{\left(a_{1}+\bar{x}_{1}\right)\left(a_{1}+x_{1}\right)}-\frac{a_{1} s_{2}}{2\left(a_{1}+x_{1}\right)\left(a_{1}+\bar{x}_{1}\right)}\right)\left(x_{1}-\bar{x}_{1}\right)^{2} \\
& -\left\{\frac{\alpha\left(a_{1}+x_{1}\right)}{a_{1} s_{1}} \times \frac{m_{1} x_{3}}{x_{2} \bar{x}_{2}}-\frac{1}{2}\left(\frac{m_{1} \alpha\left(a_{1}+x_{1}\right)}{a_{1} s_{1} \bar{x}_{2}}+\frac{m_{2}}{\bar{x}_{3}}\right)\right\}\left(x_{2}-\bar{x}_{2}\right)^{2} \\
& -\left\{\frac{m_{2} x_{2}}{x_{3} \bar{x}_{3}}+b \beta \frac{\bar{x}_{4}}{\left(a+b x_{3}+m x_{4}\right)\left(a+b \bar{x}_{3}+m \bar{x}_{4}\right)}-\frac{1}{2}\left(\frac{m_{1} \alpha\left(a_{1}+x_{1}\right)}{a_{1} s_{1} \bar{x}_{2}}+\frac{m_{2}}{\bar{x}_{3}}\right)\right. \\
& \left.-\frac{a_{1} s_{2}}{2\left(a_{1}+x_{1}\right)\left(a_{1}+\bar{x}_{1}\right)}\right\}\left(x_{3}-\bar{x}_{3}\right)^{2} \\
& -\frac{\beta\left(a+b \bar{x}_{3}\right)}{\left(a+m \bar{x}_{4}\right)} \times \frac{m \bar{x}_{3}}{\left(a+b x_{3}+m x_{4}\right)\left(a+b \bar{x}_{3}+m \bar{x}_{4}\right)}\left(x_{4}-\bar{x}_{4}\right)^{2}
\end{aligned}
$$

Since, $\frac{r}{K}>\frac{\alpha x_{2}}{\left(a_{1}+\bar{x}_{1}\right)\left(a_{1}+x_{1}\right)}+\frac{a_{1} s_{2}}{2\left(a_{1}+x_{1}\right)\left(a_{1}+\bar{x}_{1}\right)}, \quad \frac{m_{1} x_{3} \alpha\left(a_{1}+x_{1}\right)}{x_{2} \bar{x}_{2} a_{1} s_{1}}>\frac{1}{2}\left(\frac{m_{1} \alpha\left(a_{1}+x_{1}\right)}{a_{1} s_{1} \bar{x}_{2}}+\frac{m_{2}}{\bar{x}_{3}}\right), \quad \frac{m_{2} x_{2}}{x_{3} \bar{x}_{3}}+b \beta \frac{\bar{x}_{4}}{\left(a+b x_{3}+m x_{4}\right)\left(a+b \bar{x}_{3}+m \bar{x}_{4}\right)}>$ $\frac{1}{2}\left(\frac{m_{1} \alpha\left(a_{1}+x_{1}\right)}{a_{1} s_{1} \bar{x}_{2}}+\frac{m_{2}}{\bar{x}_{3}}\right)+\frac{a_{1} s_{2}}{2\left(a_{1}+x_{1}\right)\left(a_{1}+\bar{x}_{1}\right)}$, then from the above expression we conclude that $\frac{d W}{d t} \leq 0$. Hence the theorem is proved. 


\section{Optimal control}

For a better economical point of the fishery as well as the economic development of human life, we optimize the profit in bionomic equilibrium state. The bionomic equilibrium is a combination of economic equilibrium as well as biological equilibrium. Assuming that $p$ is a constant price per unit biomass, $c$ is a constant cost of harvesting effort. Then the net economic revenue calculated from the fishery is $P\left(x_{1}, x_{2}, x_{3}, x_{4}, E, t\right)=$ the total revenue obtained by selling the harvested biomass - the total cost for effort devoted to harvesting $=p q E x_{4}-c E$, then we consider an integral $J$ which is a continuous time-stream of revenues as follows:

$$
J=\int_{0}^{\infty} e^{-\delta t} P\left(x_{1}, x_{2}, x_{3}, x_{4}, E, t\right) d t,
$$

where $\delta$ denotes the instantaneous annual rate of discount [2]. Our problem is to maximize $J$ subject to the state equations (2) using Pontryagin's maximum principle [23]. The control variable $E(t)$ is subject to the constraint set, $0 \leq E \leq E_{\max }$. At first, we construct the corresponding Hamiltonian function of this optimal control problem as stated below:

$$
\begin{aligned}
H=\quad & e^{-\delta t}(p q z-c) E+\lambda_{1}\left\{r x_{1}\left(1-\frac{x_{1}}{K}\right)-\alpha \frac{x_{1} x_{2}}{a_{1}+x_{1}}-d_{1} x_{1}\right\} \\
& +\lambda_{2}\left(s_{1} \frac{x_{1} x_{2}}{a_{1}+x_{1}}+m_{1} x_{3}-m_{2} x_{2}\right) \\
& +\lambda_{3}\left(s_{2} \frac{x_{1} x_{3}}{a_{1}+x_{1}}-m_{1} x_{3}+m_{2} x_{2}-\beta \frac{x_{3} x_{4}}{a+b x_{3}+m x_{4}}\right) \\
& +\lambda_{4}\left(\beta_{1} \frac{x_{3} x_{4}}{a+b x_{3}+m x_{4}}-d_{3} x_{4}-q E x_{4}\right),
\end{aligned}
$$

where $\lambda_{i}(i=1,2,3,4)$ are called the adjoint variables.

By Pontryagin's maximum principle, the adjoint equations are described as follows:

$$
\begin{aligned}
\frac{d \lambda_{1}}{d t}=-\frac{\partial H}{\partial x_{1}}= & -\lambda_{1}\left\{r-2 \frac{r}{K} x_{1}-\frac{a_{1} \alpha x_{2}}{\left(a_{1}+x_{1}\right)^{2}}-d_{1}\right\}-\lambda_{2} a_{1} s_{1} \frac{x_{2}}{\left(a_{1}+x_{1}\right)^{2}} \\
& -\lambda_{3} \frac{a_{1} s_{2} x_{3}}{\left(a_{1}+x_{1}\right)^{2}}, \\
\frac{d \lambda_{2}}{d t}=-\frac{\partial H}{\partial x_{2}}= & \lambda_{1} \frac{\alpha x_{1}}{a_{1}+x_{1}}-\lambda_{2} \frac{s_{1} x_{1}}{a_{1}+x_{1}}+\lambda_{2} m_{2}-\lambda_{3} m_{2}, \\
\frac{d \lambda_{3}}{d t}=-\frac{\partial H}{\partial x_{3}}= & -\lambda_{2} m_{1}+\lambda_{3}\left(m_{1}+\beta x_{4} \frac{\left(a+m x_{4}\right)}{\left(a+b x_{3}+m x_{4}\right)^{2}}-\frac{s_{2} x_{1}}{a_{1}+x_{1}}\right) \\
& -\lambda_{4} \beta_{1} x_{4} \frac{\left(a+m x_{4}\right)}{\left(a+b x_{3}+m x_{4}\right)^{2}}, \\
\frac{d \lambda_{4}}{d t}=-\frac{\partial H}{\partial x_{4}}= & -p q e^{-\delta t}+\lambda_{3} \beta x_{3} \frac{\left(a+b x_{3}\right)}{\left(a+b x_{3}+m x_{4}\right)^{2}}-\lambda_{4} \beta_{1} x_{3} \frac{\left(a+b x_{3}\right)}{\left(a+b x_{3}+m x_{4}\right)^{2}} \\
& +\lambda_{4}\left(d_{3}+q E\right) .
\end{aligned}
$$

Now we derive an optimal equilibrium solution of the problem at the interior equilibrium point $\bar{P}\left(\bar{x}_{1}, \bar{x}_{2}, \bar{x}_{3}, \bar{x}_{4}\right)$. Then from equations (9) to (12), we get

$$
\begin{aligned}
& \frac{d \lambda_{1}}{d t}=\lambda_{1} A_{1}-\lambda_{2} A_{2}-\lambda_{3} A_{3}, \\
& \frac{d \lambda_{2}}{d t}=\lambda_{1} A_{4}-\lambda_{2} A_{5}-\lambda_{3} A_{6}, \\
& \frac{d \lambda_{3}}{d t}=-\lambda_{2} A_{7}+\lambda_{3} A_{8}-\lambda_{4} A_{9}, \\
& \frac{d \lambda_{4}}{d t}=-p q E e^{-\delta t}+\lambda_{3} A_{10}-\lambda_{4} A_{11} .
\end{aligned}
$$


where $A_{1}=2 \frac{r}{K} \bar{x}_{1}+\frac{a_{1} \alpha \bar{x}_{2}}{\left(a_{1}+\bar{x}_{1}\right)^{2}}+d_{1}-r, \quad A_{2}=a_{1} s_{1} \frac{\bar{x}_{2}}{\left(a_{1}+\bar{x}_{1}\right)^{2}}, \quad A_{3}=a_{1} s_{2} \frac{\bar{x}_{3}}{\left(a_{1}+\bar{x}_{1}\right)^{2}}$, $A_{4}=\alpha \frac{\bar{x}_{1}}{a_{1}+\bar{x}_{1}}, \quad A_{5}=\frac{s_{1} \bar{x}_{1}}{a_{1}+\bar{x}_{1}}-m_{2}, \quad A_{6}=m_{2}, \quad A_{7}=m_{1}, \quad A_{8}=m_{1}+\beta \bar{x}_{4} \frac{\left(a+m \bar{x}_{4}\right)}{\left(a+b \bar{x}_{3}+m \bar{x}_{4}\right)^{2}}-\frac{s_{2} \bar{x}_{1}}{a_{1}+\bar{x}_{1}}, \quad A_{9}=$ $\beta_{1} \bar{x}_{4} \frac{\left(a+m \bar{x}_{4}\right)}{\left(a+b \bar{x}_{3}+m \bar{x}_{4}\right)^{2}}, \quad A_{10}=\beta \bar{x}_{3} \frac{\left(a+b \bar{x}_{3}\right)}{\left(a+b \bar{x}_{3}+m \bar{x}_{4}\right)^{2}}, \quad A_{11}=\beta_{1} \bar{x}_{3} \frac{\left(a+b \bar{x}_{3}\right)}{\left(a+b \bar{x}_{3}+m \bar{x}_{4}\right)^{2}}+\lambda_{4}\left(d_{3}+q E\right)$.

Using the values of $\lambda_{1}, \lambda_{2}$ and $\lambda_{3}$ in equations (13) to (16), we get Hamiltonian function by which we can calculate the optimality using Pontryagin's maximum principle.

The numerical illustration of the system (2) is described in the next Section i.e., Section 6.

\section{Numerical simulation}

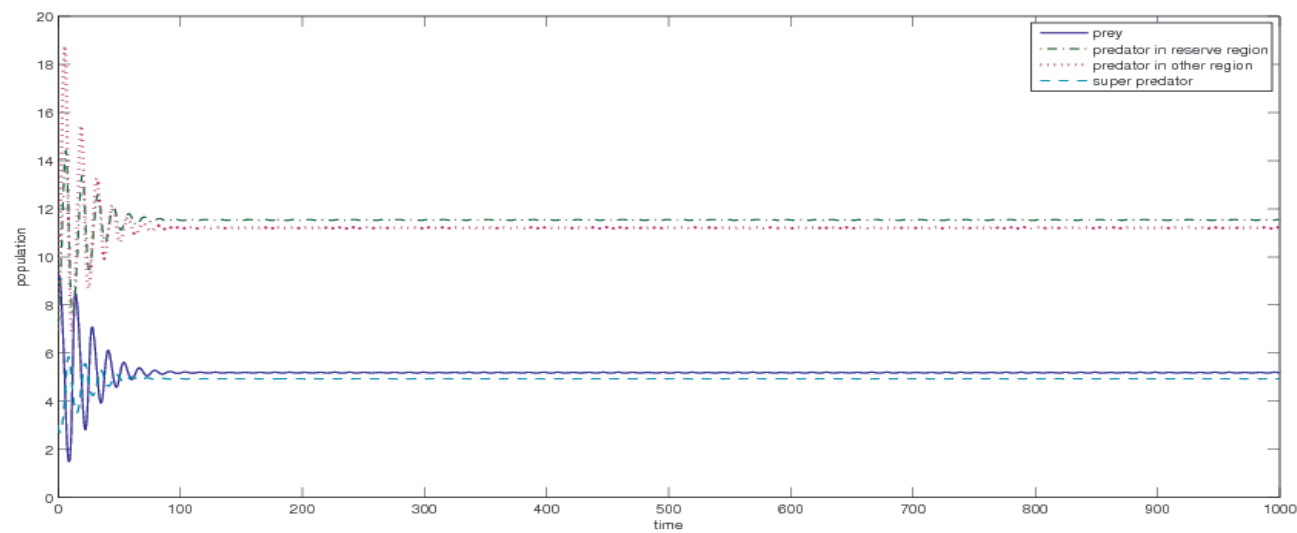

Figure 2: Graphical representation of the system (2) for $K(=14)<\bar{K}$ i.e., $K_{1}=\frac{1}{14}>\bar{K}_{1}$.

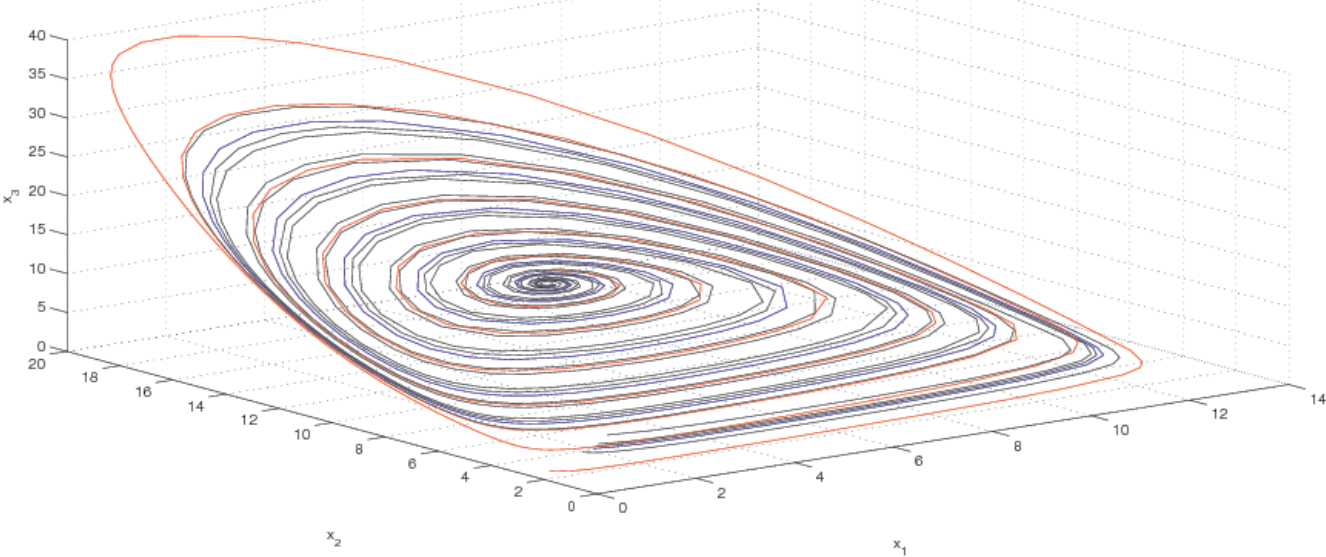

Figure 3: Phase space diagram of the system (2) with the parameter set $P_{1}$ and $K(=14)<\bar{K}$ i.e., $K_{1}=\frac{1}{14}>\bar{K}_{1}$ with respect to $x_{1}, x_{2}$ and $x_{3}$. 


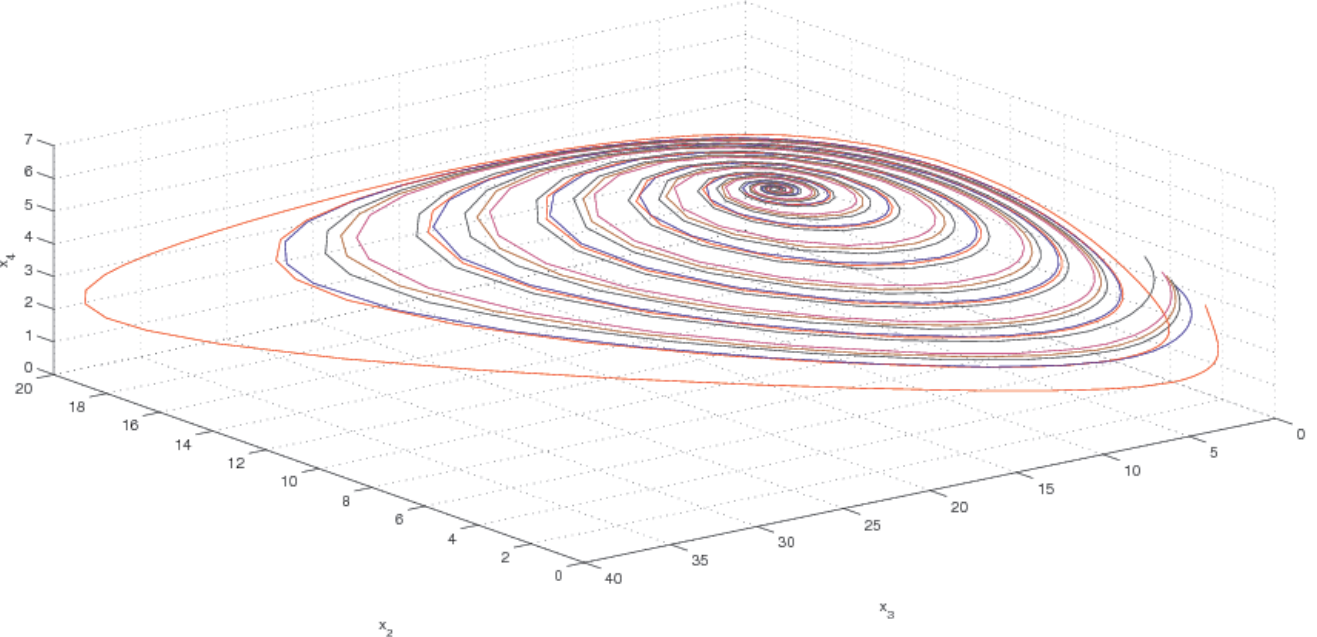

Figure 4: Phase space diagram of the system (2) with the parameter set $P_{1}$ and $K(=14)<\bar{K}$ i.e., $K_{1}=\frac{1}{14}>\bar{K}_{1}$ with respect to $x_{2}, x_{3}$ and $x_{4}$.

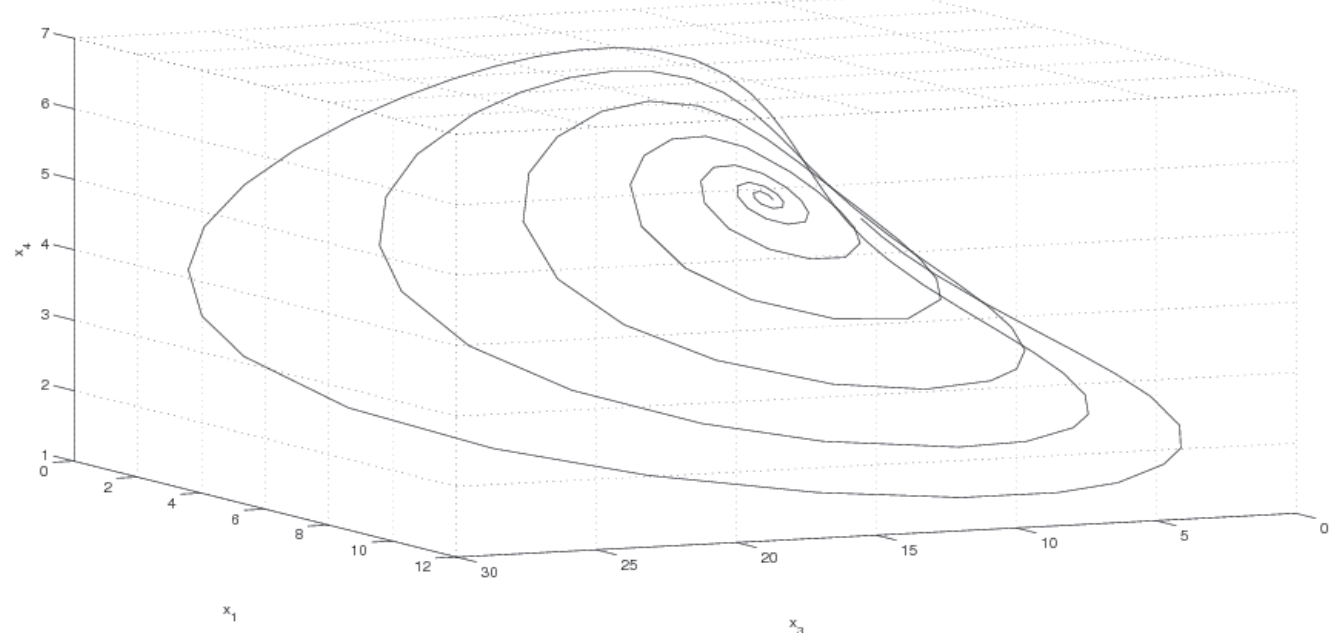

Figure 5: Phase space diagram of the system (2) with the parameter set $P_{1}$ and $K(=14)<\bar{K}$ i.e., $K_{1}=\frac{1}{14}>\bar{K}_{1}$ with respect to $x_{1}, x_{3}$ and $x_{4}$.

Describing the discussed phenomenon with their relative factors, we justify the analytical results of the proposed system with the hypothetical data set. The results of the simulation experiment are taken using MATLAB 7.10. Choosing the parameter set arbitrarily as $P_{1}=\left\{r=2.5, \alpha=1.3, a_{1}=5, d_{1}=0.03, m_{1}=\right.$ $\left.0.2, m_{2}=0.5, s_{1}=0.6, s_{2}=0.6, \beta=0.8, \beta_{1}=0.2, d_{3}=0.2, a=5, b=0.1, m=0.05, q=0.2, E=0.76\right\}$. Now, $K$ can be taken as (i) $K(=14)<\bar{K}$ and (ii) $K(=16)>\bar{K}$.

We consider two cases as follows:

Case (i), we draw Figures 2, 3, 4 and 5 by choosing the parameter set $P_{1}$. From Figure 2, we see that the proposed system is stable after certain time. Figure 3 shows the phase space diagram of the system with 


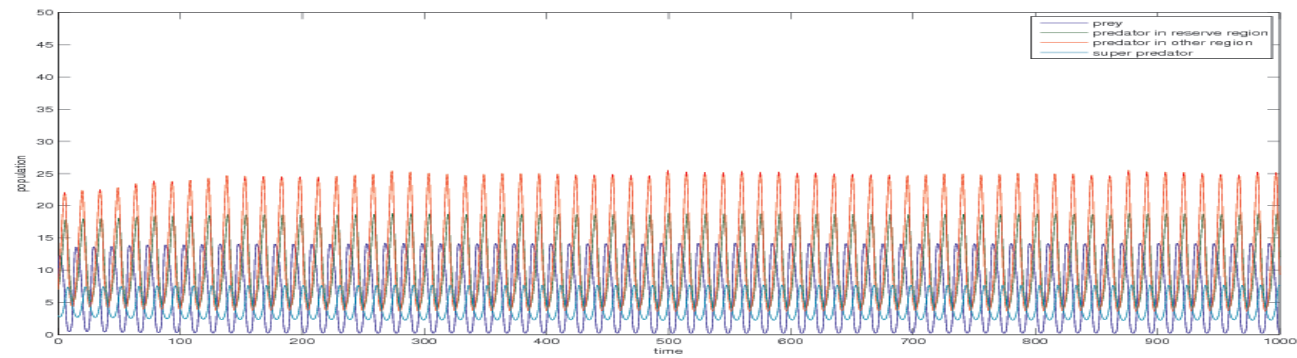

Figure 6: Graphical representation of the system (2) for $K(=16)>\bar{K}$ i.e., $K_{1}=\frac{1}{16}<\bar{K}_{1}$.



Figure 7: Phase space diagram of the system (2) with the parameter set $P_{1}$ and $K(=16)>\bar{K}$ i.e., $K_{1}=\frac{1}{16}<\bar{K}_{1}$ with respect to $x_{1}, x_{2}$ and $x_{3}$.

respect to $x_{1}, x_{2}$ and $x_{3}$. Similarly, Figures 4 and 5 show the phase diagram of the system (2) with respect to $x_{2}, x_{3}, x_{4}$ and $x_{1}, x_{3}, x_{4}$, respectively.

Case (ii), we depict Figures 6, 7, 8 and 9 by taking the parameter set $P_{1}$. From Figure 6, we observe that the proposed system is unstable. Figure 7 shows the phase space diagram of the system with respect to $x_{1}, x_{2}$ and $x_{3}$. Similarly, Figures 8 and 9 show the phase diagram of the system (2) with respect to $x_{2}, x_{3}, x_{4}$ and $x_{1}, x_{3}, x_{4}$, respectively. Figure 10 shows the bifurcation diagram of the system (2) with the parameter set $P_{1}$. For existence of interior equilibrium point $P\left(\bar{x}_{1}, \bar{x}_{2}, \bar{x}_{3}, \bar{x}_{4}\right)$, the conditions $r>d_{1}, \beta_{1}>b\left(d_{3}+q E\right)$ will hold. The parameter set $P_{1}$ and $K=14$ satisfy these conditions. By choosing the parameter set $P_{1}$, Figure 2 is drawn which shows stable equilibrium of the system. Now we consider $r(=2.5)<d_{1}(=3)$ and then draw Figure 11, which shows unstable equilibrium. Again, considering $P_{2}=\left\{r=2.5, \alpha=1.3, a_{1}=5, d_{1}=3, m_{1}=\right.$ $\left.0.2, m_{2}=0.5, s_{1}=0.6, s_{2}=0.6, \beta=0.8, \beta_{1}=0.2, d_{3}=0.2, a=5, b=1, m=0.05, q=0.2, E=0.76\right\}$ as the set of parameters, where $\beta_{1}<b\left(d_{3}+q E\right)$, we depict Figure 12. From Figures 11 and 12, it is easily seen that some populations become endanger species. 


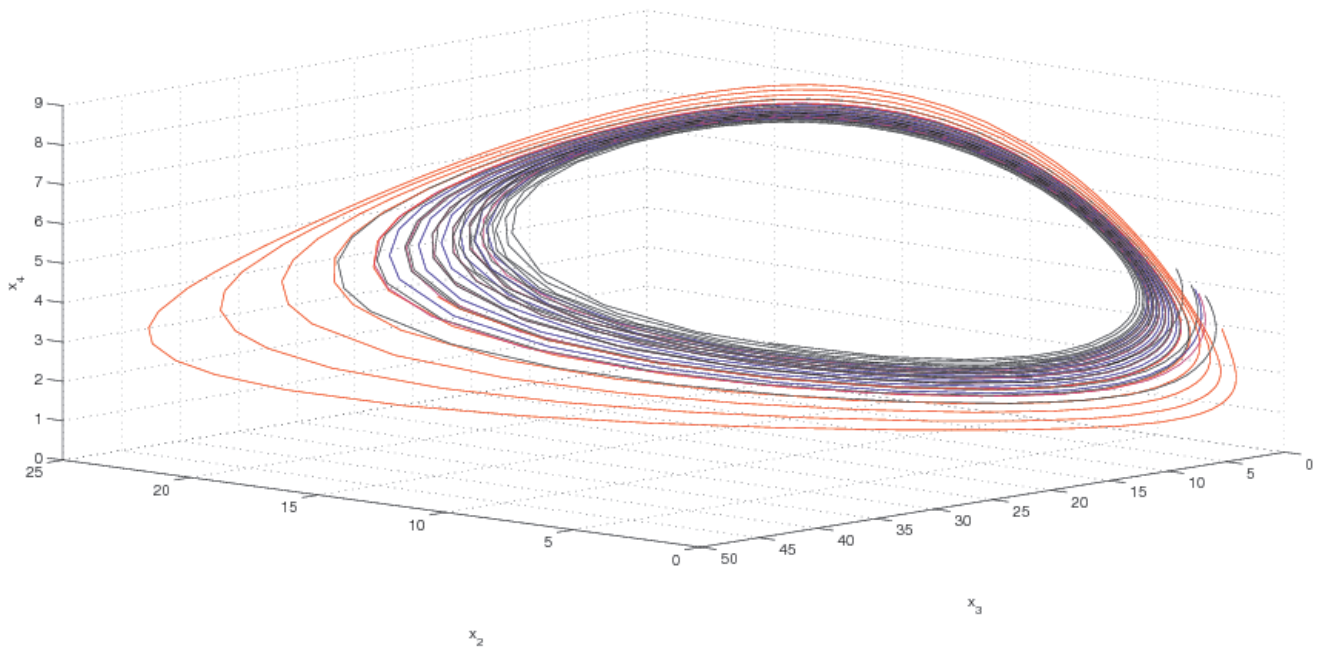

Figure 8: Phase space diagram of the system (2) with the parameter set $P_{1}$ and $K(=16)>\bar{K}$ i.e., $K_{1}=\frac{1}{16}<\bar{K}_{1}$ with respect to $x_{2}, x_{3}$ and $x_{4}$.



Figure 9: Phase space diagram of the system (2) with the parameter set $P_{1}$ and $K(=16)>\bar{K}$ i.e., $K_{1}=\frac{1}{16}<\bar{K}_{1}$ with respect to $x_{1}, x_{3}$ and $x_{4}$.

\section{Conclusion}

In this paper, we have formulated the mathematical model on prey-predator relationship in a drainage basin. To the best of our knowledge, the presented work is the first attempt to consider reserve region for the predator population. Based on the discussed phenomena, we have considered a food chain with a reserve region of predator. A study of potential effects of generalist predator in a predator and predator in a prey has been introduced with Beddington-DeAngelis functional response and Holling type II functional response, respectively. The density-dependent mortality rates for prey and generalist predator have been assumed. Depending on different environments, we have considered different consumption rates of predator populations. The equilibria of the proposed system have been determined with the discussions on local and global stability for the 

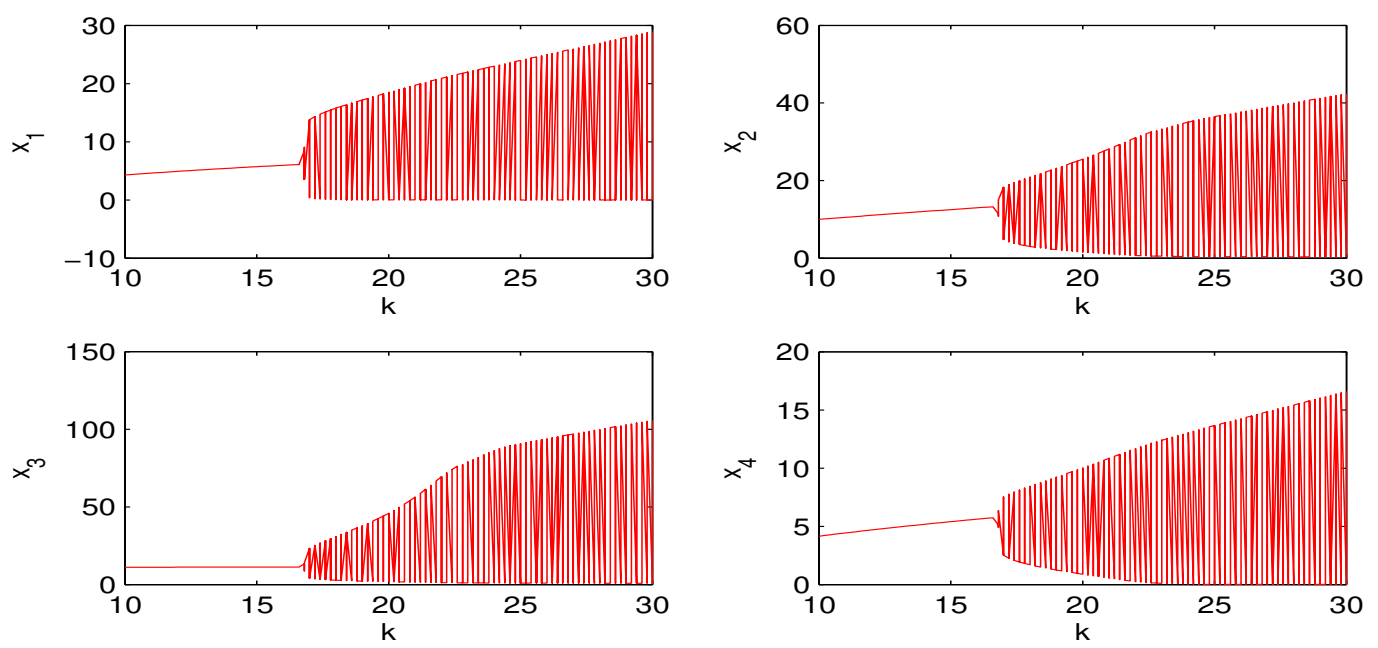

Figure 10: Bifurcation diagram of the system (2) with the parameter set $P_{1}$.

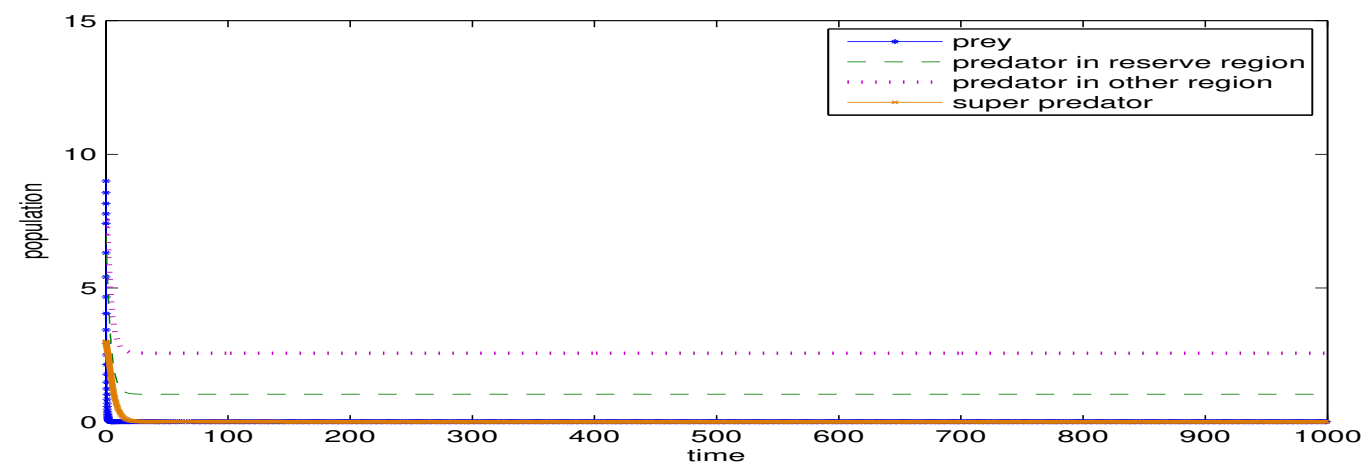

Figure 11: Graphical representation of the system (2) with parameter set $P_{2}$ and $K=14$ for $r(=2.5)<d_{1}(=3)$.

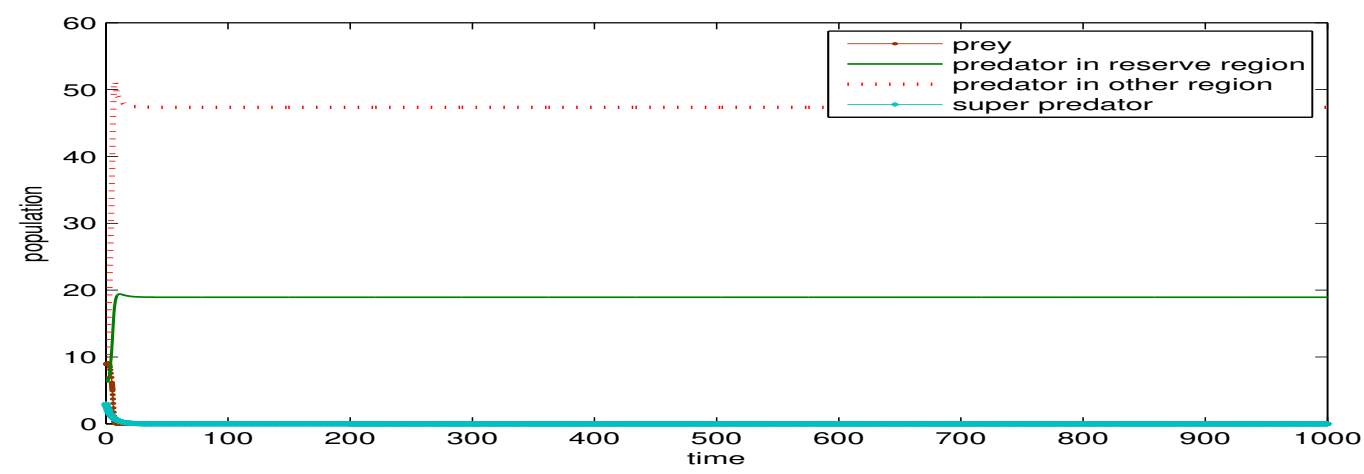

Figure 12: Graphical representation of the system (2) with parameter set $P_{2}$ and $K=14$. 
system. We optimize the profit in bionomic equilibrium state. With the help of this control parameter, a dynamic framework is developed to investigate the optimal utilization of resources, sustainability properties of the stock and the resource rent. Existence criteria at interior equilibrium point and stability of the proposed model has been discussed in numerical simulation and analyzed through the geometrical figures.

The main theoretical results have been demonstrated by numerical simulation with hypothetical parameter values. Someone can verify the validity of the system by considering real data sets. Mosquito-borne diseases control model can be developed for reducing mosquito larva. Hence, future research work is necessary to achieve the needs in this direction.

Acknowledgement: The authors are very much thankful to the Editor-in-Chief and anonymous reviewers for their precious comments that helped us to rigorously improve the quality of the manuscript.

Compliance with ethical standards

Conflict of interest: The authors do not have any possible conflicts of interest.

Ethical approval: This article does not contain any studies with human participants or animals performed by any of the authors.

Data Availability Statement: Data sharing is not applicable to this article as no datasets were generated or analysed during the current study.

\section{References}

[1] A. Lotka, Elements of Physical Biology, Williams and Wilkins, Baltimore, 1925.

[2] D. Purohit, K.S. Chaudhuri, A Bioeconomic model of nonselective harvesting of two competing fish species, ANZIAMJ 46 (2004) 299-308.

[3] B. Roy, S.K. Roy, Analysis of prey-predator three species models with vertebral and invertebral predators, International Journal Dynamics and Control 3 (2015) 306-312.

[4] H. Kharbanda, S. Kumar, Chaos detection and optimal control in a cannibalistic prey-predator system with harvesting, International Journal of Bifurcation and Chaos 30 (2020) 2050171.

[5] K. Chakraborty, T.K. Kar, S. Jana, Global dynamics and bifurcation in a stage structured prey-predator fishery model with harvesting, Applied Mathematics and Computation 218 (2012) 9271-9290.

[6] J. P. Tripathi, S. Tyagi, S. Abbas, Dynamical analysis of a predator-prey interaction model with time delay and prey refuge, Nonautonomous Dynamical Systems 5 (2018) 138-151.

[7] B. Roy, S. K. Roy, M. H. A. Biswas, Effects on prey-predator with different functional responses, International Journal of Biomathematics 10 (2017) 1750113 (22 pages).

[8] A. Jana, S. K. Roy, Holling-Tanner prey-predator model with Beddington-DeAngelis functional response including delay, International Journal of Modelling and Simulation (2020) DOI: 10.1080/02286203.2020.1839168.

[9] H. Qiu, S. Guo, S. Li , Stability and bifurcation in a predator-prey system with prey-taxis, International Journal of Bifurcation and Chaos 30 (2020) 2050022.

[10] J.P. Tripathi, S. S. Meghwani, S. Tyagi, S. Abbas, Global dynamics and parameter identifiability in a predator-prey interaction model, Nonautonomous Dynamical Systems 5 (2018) 113-126.

[11] R. Banerjee, P. Das, D. Mukherjee, Stability and permanence of a discrete-time two-prey one-predator system with Holling Type-III functional response, Chaos, Solitons and Fractals 117 (2018) 240-248.

[12] S. Kumar, H. Kharbanda, Chaotic behavior of predator-prey model with group defense and non-linear harvesting in prey, Chaos, Solitons and Fractals 119 (2019) 19-28.

[13] W. Abid, R. Yafia, M. A. Aziz-Alaoui, A. Aghriche, Dynamics analysis and optimality in selective harvesting predator-prey model with modified Leslie-Gower and Holling-Type II, Nonautonomous Dynamical Systems 6 (2019) 1-17.

[14] C.W. Clark, Mathematical bioeconomics: the optimal management of renewable resources, Wiley, New York, 1976.

[15] T. Das, R.N. Mukherjee, K.S. Chaudhuri, Harvesting of a prey-predator fishery in the presence of toxicity, Applied Mathematical Modelling 33 (2009) 2282-2292.

[16] B. Roy, S. K. Roy, D. B. Gurung, Holling-Tanner model with Beddington-DeAngelis functional response and time delay introducing harvesting, Mathematics and Computers in Simulation 142 (2017) 1-14.

[17] B. Ghosh, T.K. Kar, Sustainable use of prey species in a prey-predator system: Jointly determined ecological thresholds and economic trade-offs, Ecological Modelling 272 (2014) 49-58. 
[18] M. Sen, A. Simha, S. Raha, Adaptive control based harvesting strategy for a predator-prey dynamical system, Acta Biotheoretica 66 (2018) 293-313.

[19] S. Jana, M. Chakraborty, K. Chakraborty, T.K. Kar, Global stability and bifurcation of time delayed prey-predator system incorporating prey refuge, Mathematics and Computers in Simulation 85 (2012) 57-77.

[20] S.K. Roy, B. Roy, Analysis of prey-predator three species fishery model with harvesting including prey refuge and migration, International Journal of Bifurcation and Chaos 26 (2016) 1650022.

[21] Y. Li, J. Li, Discrete-time models for releases of sterile mosquitoes with Beverton-Holt-type of survivability, Ricerche di Matematica 67 (2018) 141-162.

[22] V. Venkatsubramanian, H. Schattler, J. Zaborszky, Local bifurcation and feasibility regions in differential-algebraic systems, IEEE Transactions on Automatic Control 12 (1995) 1992-2013.

[23] L.S. Pontryagin, V.S. Boltyanskii, R.N. Gamkrelidge, E.F. Mishchenko, The mathematical theory of optimal processes, Wiley, New York, 1962. 